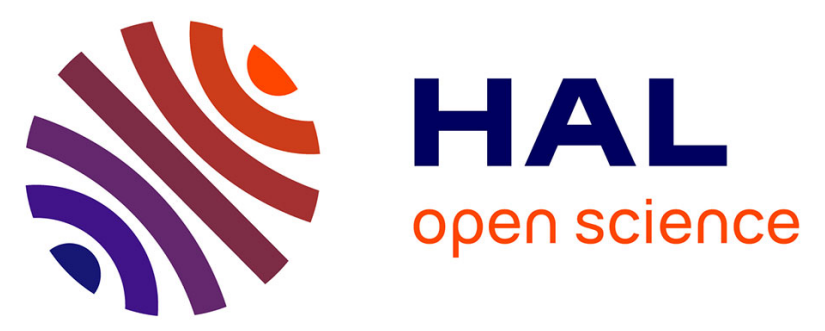

\title{
Optical transmission properties of an anisotropic defect cavity in one-dimensional photonic crystal
}

\author{
Noama Ouchani, Abdelaziz El Moussaouy, Hassan Aynaou, Youssef El \\ Hassouani, El Houssaine El Boudouti, Bahram Djafari-Rouhani
}

\section{To cite this version:}

Noama Ouchani, Abdelaziz El Moussaouy, Hassan Aynaou, Youssef El Hassouani, El Houssaine El Boudouti, et al.. Optical transmission properties of an anisotropic defect cavity in one-dimensional photonic crystal. Physics Letters A, 2018, 382 (4), pp.231-240. 10.1016/j.physleta.2017.11.020 . hal03183514

\author{
HAL Id: hal-03183514 \\ https://hal.science/hal-03183514
}

Submitted on 26 Aug 2021

HAL is a multi-disciplinary open access archive for the deposit and dissemination of scientific research documents, whether they are published or not. The documents may come from teaching and research institutions in France or abroad, or from public or private research centers.
L'archive ouverte pluridisciplinaire HAL, est destinée au dépôt et à la diffusion de documents scientifiques de niveau recherche, publiés ou non, émanant des établissements d'enseignement et de recherche français ou étrangers, des laboratoires publics ou privés.

\section{(c)(1)}

Distributed under a Creative Commons Attribution| 4.0 International License 


\title{
Optical transmission properties of an anisotropic defect cavity in one-dimensional photonic crystal
}

\author{
Noama Ouchani ${ }^{a, *}$, Abdelaziz El Moussaouy ${ }^{\mathrm{b}, \mathrm{c}}$, Hassan Aynaou ${ }^{\mathrm{d}}$, Youssef El Hassouani ${ }^{\mathrm{e}}$, \\ El Houssaine El Boudouti ${ }^{\mathrm{f}}$, Bahram Djafari-Rouhani ${ }^{\mathrm{g}}$
}

a Centre Régional des Métiers de l'Education et de la Formation, 30000 Fès, Morocco

b LDOM, Département de Physique, Faculté des Sciences, Université Mohamed I, 60000 Oujda, Morocco

c Centre Régional des Métiers de l'Education et de la Formation, 60000 Oujda, Morocco

d EPSMS, Département de Physique, Faculté des Sciences et Techniques, Université Moulay Ismail, Boutalamine BP 509, 52000 Errachidia, Morocco

e ESIM, Département de Physique, Faculté des Sciences et Techniques, Université Moulay Ismail, Boutalamine BP 509, 52000 Errachidia, Morocco

f LPMR, Département de Physique, Faculté des Sciences, Université Mohammed Premier, 60000 Oujda, Morocco

g IEMN, UMR-CNRS 8520, UFR de Physique, Université de Lille 1, 59655 Villeneuve d'Ascq, France

We investigate theoretically the possibility to control the optical transmission in the visible and infrared regions by a defective one dimensional photonic crystal formed by a combination of a finite isotropic superlattice and an anisotropic defect layer. The Green's function approach has been used to derive the reflection and the transmission coefficients, as well as the densities of states of the optical modes. We evaluate the delay times of the localized modes and we compare their behavior with the total densities of states. We show that the birefringence of an anisotropic defect layer has a significant impact on the behavior of the optical modes in the electromagnetic forbidden bands of the structure. The amplitudes of the defect modes in the transmission and the delay time spectrum, depend strongly on the position of the cavity layer within the photonic crystal. The anisotropic defect layer induces transmission zeros in one of the two components of the transmission as a consequence of a destructive interference of the two polarized waves within this layer, giving rise to negative delay times for some wavelengths in the visible and infrared light ranges. This property is a typical characteristic of the anisotropic photonic layer and is without analogue in their counterpart isotropic defect layers. This structure offers several possibilities for controlling the frequencies, transmitted intensities and the delay times of the optical modes in the visible and infrared regions. It can be a good candidate for realizing high-precision optical filters.

\section{Introduction}

Photonic Crystals (PCs) have attracted a great deal of attention during the last two decades [1-3] due to their potential applications in optoelectronic and optical communications [4-16]. They can be used in the fabrication of lasers [4,5], optical diodes [6,7], waveguides [8,9], filters [10], dielectric reflectors [11-14], sensors $[15,16]$, etc. Research in PCs has known a tremendous expansion and covers a wide range of electromagnetic spectrum from microwaves to the visible. Several technological difficulties restrict the fabrication of three-dimensional (3D) PCs in the visible (VIS) and infrared (IR) regions, due to their small lattice constant, which should be comparable to the wavelength [17]. However, the com-

\footnotetext{
* Corresponding author.

E-mail address: noama03@yahoo.fr (N. Ouchani).
}

plication associated with 3D PCs lead to the investigation of onedimensional (1D) periodic structures, which can be easily produced for this range of wavelengths, by using thin-film deposition techniques.

For most applications, a defective PCs are more desired than the perfect ones. The physical properties of the localized or defect modes within the photonic band gaps (PBGs) has been increasingly studied. According to such combination, several materials are used as the defect layer in the design of the photonic devices. Several works have investigated the optical and microwave properties of defect modes in 1D isotropic dielectric PCs. Liu et al. [10] have studied a 1D PC structure consisting of alternate layers of $\mathrm{Ta}_{2} \mathrm{O}_{5}$ and $\mathrm{MgF}_{2}$ films with a defect layer in the visible region. They have shown that the number and the frequencies of the defect modes can be controlled in the visible range by adjusting the thickness of the defect layer. This structure can be useful in the design of blue- 


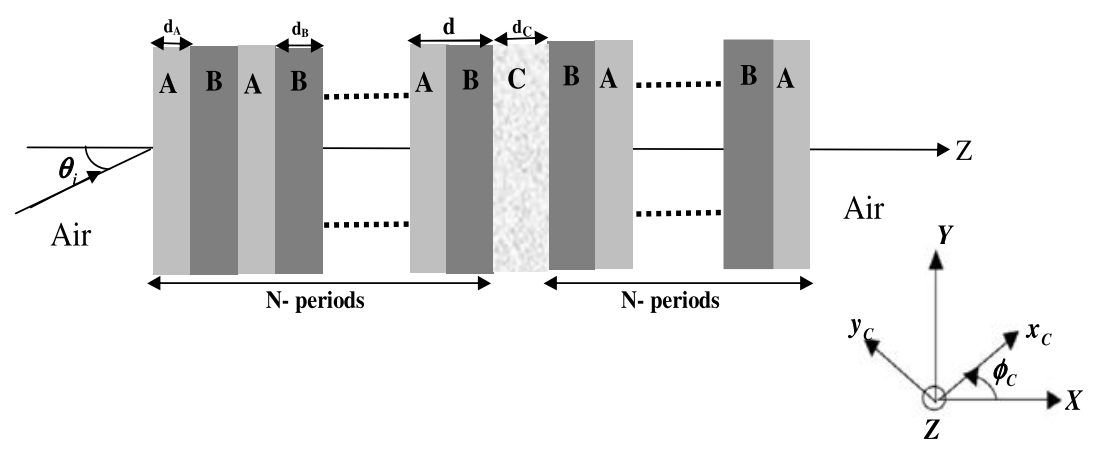

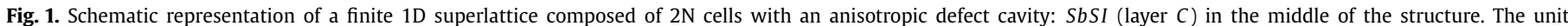

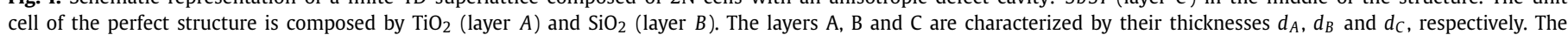

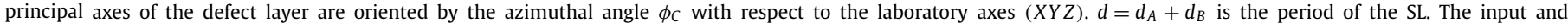
output isotropic media are air and the incident electromagnetic wave is launched with an angle $\theta_{i}$ with respect to the normal to the superlattice.
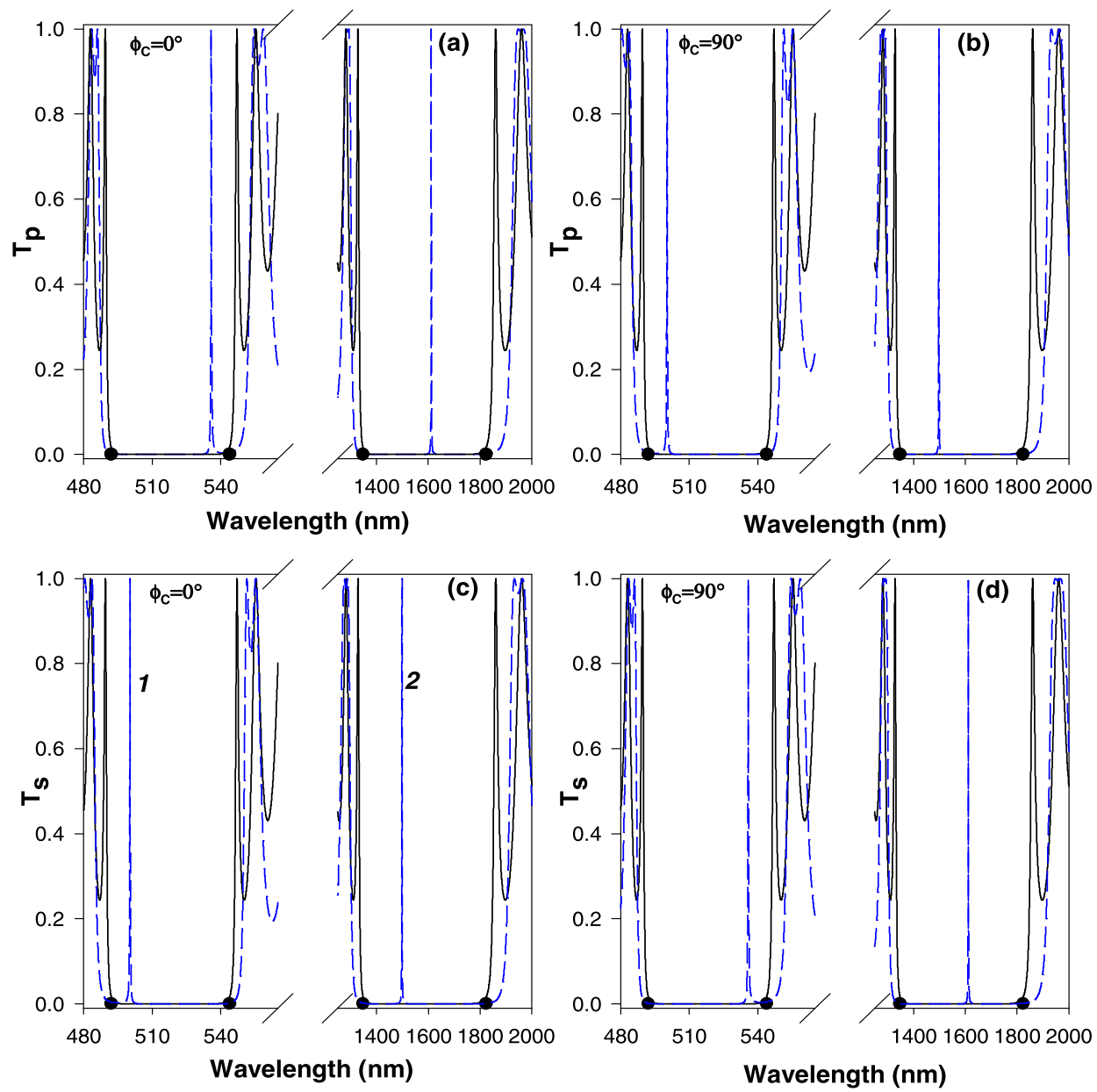



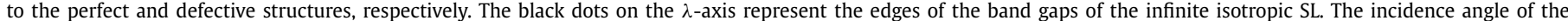
incoming light is $\theta_{i}=0^{\circ}$.

green color filters. The green and red color filters were studied by Xiang et al. [18].

Superconductor material and liquid crystal are among potential materials to serve as defect layers in tunable photonic devices, as their optical properties can be controlled by external excitation. Indeed, $\mathrm{Wu}$ and Gao [16] have studied the effect of the temperature on the defect modes in a 1D dielectric PC heterostructure with a superconducting defect. They have shown that this structure has very high temperature sensitivity. Dadoenkova et al. [19] have investigated PBG spectra of a 1D dielectric PC with a complex defect layer, consisting of ultrathin superconducting and dielectric sublayers. Recently, some research works [20-23] have investigated the effect of an electric field on the defect modes induced by a liquid crystal defect layer (anisotropic medium) or by an $\mathrm{Ag} / \mathrm{LiNbO}_{3} / \mathrm{Ag}$ sandwich structure into 1D PC [22,23]. Tang el al. [24] have explored the influence of the anisotropy of the singlenegative material on photonic band gaps and tunneling modes of 1D PCs containing dispersive anisotropic single-negative metamaterials. Penninck et al. [25] have studied the emission of light in uniaxially anisotropic thin film devices. This emission is performed 

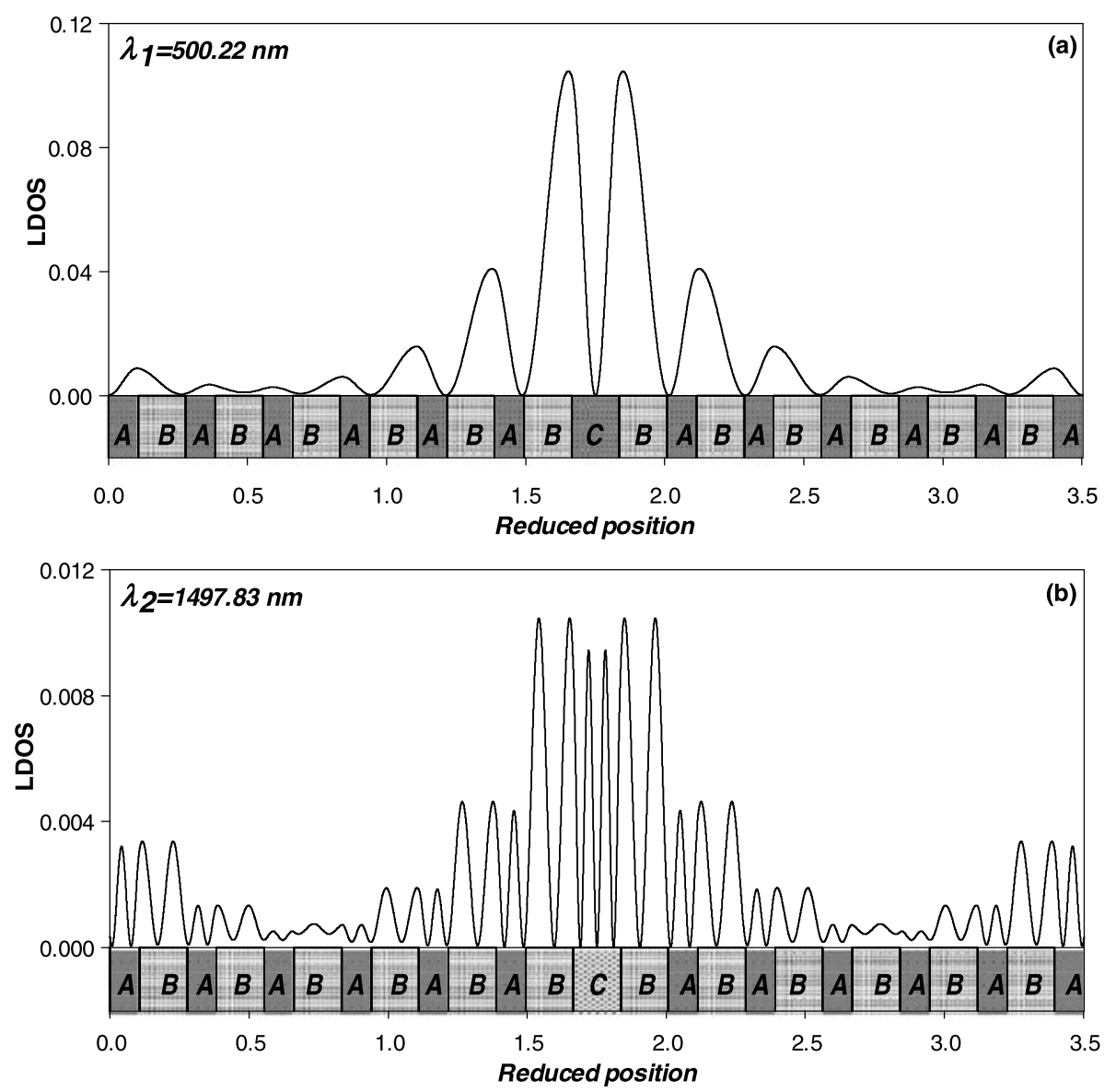

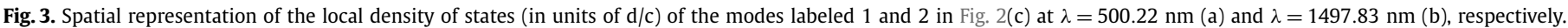
The input $S$-polarized wave is launched normally to the SL and the azimuthal angle of the layer $C$ is $\phi_{C}=0^{\circ}$.

through a dipole antenna inserted inside a thin isotropic film sandwiched between two anisotropic layers. The whole structure is placed between glass and air.

1D birefringent photonic crystal based on anisotropic porous silicon layers has been designed to control simultaneous enhancement of second and third harmonic generation [26]. The structure consists of 12 quarter wavelength pairs of layers separated by a half wavelength cavity spacer. Recently the chiral PCs with defects have been of particular interest due to their rich optical properties. Various types of defects have been considered, such as: a thin isotropic [27-29] or anisotropic [30-32] defect layers inserted between two chiral PCs. The properties of the defect modes induced by anisotropic dielectric layer in 1D birefringent photonic crystal, have been investigated theoretically by some of us [33].

In all the above works, only the reflectance and/or the transmittance of defect modes have been explored. In this work, we are interested to some optical transmission properties of the defect modes such as: the transmission delay times and the behavior of total densities of states (DOS) in the visible and infrared regions of a defective one dimensional photonic crystal constituted by a combination of a finite isotropic superlattice (SL) and a dielectric anisotropic defect layer. In particular, we analyze the delay times of the defect modes and compare their behavior with the total DOS. More especially, we show the possibility of existence of transmission zeros induced by the anisotropic defect layer, giving rise to negative delay times for some wavelengths in the visible and infrared regions. Let us recall that the existence of transmission zeros has been shown in 2D photonic crystals [34,35], however the possibility of existence of such forbidden transmission regions in 1D lamellar systems has not been investigated before. This property is a characteristic of the anisotropic defect layer and is without analogue in their counterpart isotropic layer. It is worth mentioning that transmission zeros and the corresponding negative delay times have been demonstrated in quasi-one dimensional circuits made of coaxial cables in the radio-frequency domain [36]. Also, we highlight the impact of the orientation of the principal axes of the defect layer with respect to the laboratory axes, on the behavior of the optical modes in the forbidden band-gap of the system. The effect of the position of the cavity layer within the photonic crystal on the transmittance and the delay time of the defect modes will be also investigated. In general, the main theoretical method adopted for the analysis of the 1D multilayered PCs, is the transfer matrix method $[10,12,14]$. In this paper, we use the Green's function approach which enables to derive the transmission and reflection coefficients, the dispersion curves, the delay times as well as the total and local DOS of electromagnetic modes propagating through the structure.

This paper is organized as follows: in section 2 we present the theoretical model and the method to derive the expressions of the reflection and transmission coefficients, the delay time and the densities of states, when $S$ or $P$ polarized waves is incident upon the interface separating the substrate and the SL. Section 3 gives the numerical results of the transmittance and the delay time of the optical modes with respect to the wavelength and their dependence on the azimuthal angle and the position of the defect layer within the photonic crystal. The spatial behavior of electromagnetic modes along the $z$-axis of the SL, has been investigated through the local density of states. Finally, a summary of this work is presented in section 4 . 



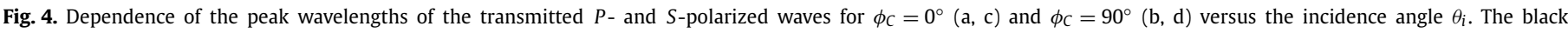
triangles and dots denote the defect modes in PBGs. The dotted curves represent the edges of the band gaps (hatched areas) of the infinite isotropic SL.

\section{Theoretical model}

The geometrical structure is schematically illustrated in Fig. 1. It is denoted by $s_{1} /(A B)^{N} C(B A)^{N} / s_{2}$, where $s_{i}(i=1$ or 2$)$ means the semi-infinite substrates and $N$ is the number of the $A B$ bilayers (periods) at each side of the anisotropic defect layer $C$. The unit cell of the structure is composed of two dielectric isotropic layers $A$ and $B$. All the interfaces of the layers are taken to be parallel to the (XY) plane of a cartesian (laboratory) coordinate system and the $\mathrm{Z}$ axis is along the normal to the interfaces (Fig. 1). The thicknesses of the layers $A, B$ and $C$ are denoted $d_{A}, d_{B}$ and $d_{C}$, respectively. $d=d_{A}+d_{B}$ is the period of the SL. The materials constituting the layers are assumed to be homogeneous and non-magnetic. The isotropic layers $A$ and $B$ are characterized by the dielectric constants $\epsilon_{A}$ and $\epsilon_{B}$ respectively, whereas the anisotropic layer is characterized by the diagonal dielectric tensor $\epsilon=\left(\epsilon_{x}, \epsilon_{y}, \epsilon_{z}\right)$, where $\epsilon_{x}, \epsilon_{y}$ and $\epsilon_{z}$ are the dielectric constants in the corresponding principal axes (xyz) of the crystal. For a given orientation of the crystal, its dielectric tensor with respect to the fixed (XYZ) coordinate system is described by using the Euler angles $[37,38] \theta, \phi$ and $\psi$. For the sake of simplicity and without loss of generality, we investigate in this study the effect of only the azimuthal angle $\phi$ and omit the effect of the two other angles $(\theta=\psi=0)$. The axes of the birefringent layer $C$ are shown in Fig. 1.

Among different techniques used to study the propagation of electromagnetic waves in periodic structures, one can cite the transfer matrix [39,40] and the Green's function methods [41]. Both techniques enable to calculate dispersion relations and transmission and reflection coefficients. However, the Green's function presents the advantage to deduce easily the densities of states as well as different scattering properties of the system [41]. An interesting connection between Green's functions and transfer matrices has been established by Velasco et al. [42] and El Boudouti et al. [43]. This combination yields to study of the physical properties of any composite system. In this work, we use a simple formulation of the Green's function called interface response theory of continuous media [44]. This technique is suitable for treating composite systems containing a large number of interfaces [41]. In this theory, the Green's function of a composite system can be written as [44]

$$
\begin{aligned}
g(D D)= & G(D D)+G(D M)\left\{[G(M M)]^{-1} g(M M)[G(M M)]^{-1}\right. \\
& \left.-[G(M M)]^{-1}\right\} G(M D),
\end{aligned}
$$

where $D$ and $M$ are respectively, the whole space and the space of the interfaces in the lamellar system. $G$ is a block-diagonal matrix in which each block $G_{i}$ corresponds to the bulk Green's function of the subsystem $i$. All the matrix elements $g(D D)$ of the composite material can be obtained from the knowledge of the matrix elements $g(M M)$ in the interface space $M . g(M M)$ is calculated from its inverse $g^{-1}(M M)$. The latter is formed out by a linear superposition of the surface matrix elements $g_{i}^{-1}(M M)$ of any independent film bounded by perfectly free interfaces with appropriate boundary conditions. The matrix elements $g_{i}^{-1}(M M)$ for an anisotropic medium are given in previous works $[33,45]$.

Within this theory, the reflected and transmitted waves $u(D)$, resulting from a uniform plane wave $U(D)$ incident upon a plane boundary between two different media, are given by [44] 

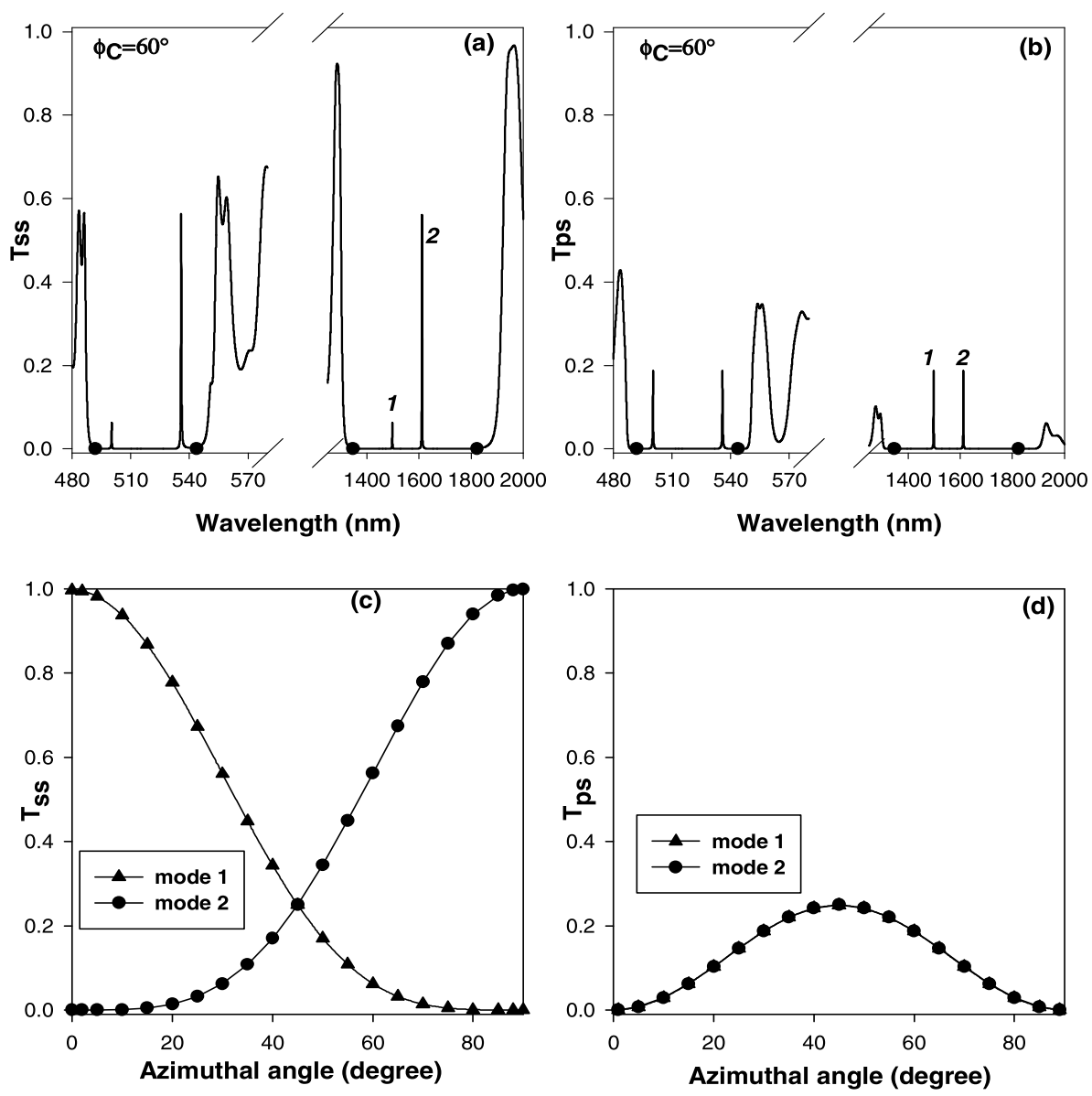

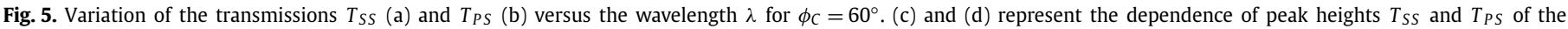
defect modes labeled 1 and 2 in (a) and (b) on the azimuthal angle $\phi_{C}$, respectively. The incoming $S$-polarized light is launched normally to the SL.

$$
\begin{aligned}
u(D)= & U(D)+G(D M)\left\{[G(M M)]^{-1} g(M M)[G(M M)]^{-1}\right. \\
& \left.-[G(M M)]^{-1}\right\} U(M) .
\end{aligned}
$$

Let us mention that the incident wave, generated in the substrate $s$, can have two different polarizations, namely, $S$ or $P$. In general, each wave entering the anisotropic medium, generates two transmitted waves and two reflected waves with different polarizations. Let us call $\mathrm{E}_{i S}$ and $\mathrm{E}_{i P}$ the amplitudes of the $S$ and $P$ components of the incident field respectively. Then, the amplitudes of the reflected and transmitted fields can be written as $[33,45]$ :

$$
\begin{aligned}
& \mathbf{E}_{R S}(z)=r_{S S} \mathbf{E}_{i S}(z)+r_{S P} \mathbf{E}_{i P}(z), \\
& \mathbf{E}_{T S}(z)=t_{S S} \mathbf{E}_{i S}(z)+t_{S P} \mathbf{E}_{i P}(z), \\
& \mathbf{E}_{R P}(z)=r_{P S} \mathbf{E}_{i S}(z)+r_{P P} \mathbf{E}_{i P}(z), \\
& \mathbf{E}_{T P}(z)=t_{P S} \mathbf{E}_{i S}(z)+t_{P P} \mathbf{E}_{i P}(z) .
\end{aligned}
$$

The expressions of $r_{i j}$ and $t_{i j}$ in these equations $(i, j=S$ or $P)$ for an anisotropic structure are given in the previous works $[33,45]$. Therefore, the total reflection coefficients for the $P$ and $S$ modes are given respectively by

$R_{P}=R_{P P}+R_{S P}=\left|r_{P P}\right|^{2}+\left|r_{S P}\right|^{2}$,
$R_{S}=R_{S S}+R_{P S}=\left|r_{S S}\right|^{2}+\left|r_{P S}\right|^{2}$,

and the total transmission coefficients for the $P$ and $S$ modes are given respectively by

$T_{P}=T_{P P}+T_{S P}=\left|t_{P P}\right|^{2}+\left|t_{S P}\right|^{2}$,

$$
T_{S}=T_{S S}+T_{P S}=\left|t_{S S}\right|^{2}+\left|t_{P S}\right|^{2} .
$$

From the expressions of the $r_{i j}$ and $t_{i j}$, one can deduce also the phases of the transmission and reflection coefficients. The derivatives of these phases with respect to the pulsation $\omega$, yield to an important physical quantity. This latter called the delay time, indicates the times needed by the wave to complete the transmission or reflection process through the structure. Four different delay times can be defined for the transmission coefficients, namely

$\tau_{s s}=\frac{d \phi_{s s}}{d \omega} ; \quad \tau_{p s}=\frac{d \phi_{p s}}{d \omega} ; \quad \tau_{p p}=\frac{d \phi_{p p}}{d \omega} ; \quad \tau_{s p}=\frac{d \phi_{s p}}{d \omega}$

where $\phi_{s s}, \phi_{p s}, \phi_{p p}, \phi_{s p}$ are respectively the phases of the transmission coefficients $t_{s s}, t_{p s}, t_{p p}, t_{s p}$. As mentioned above, the Green's function enables to calculate the density of states, especially, one can determine the variation of the density of states $\Delta n$ between the structure $s_{1} /(A B)^{N} C(B A)^{N} / s_{2}$ and a reference system formed out of the same volumes of the bulk semi-infinite substrates $s_{1}$ and $s_{2}$ and the finite SL. This quantity is given by [46]:

$\Delta n(\omega)=\frac{1}{\pi} \frac{d}{d \omega} \arg \left\{\operatorname{det}\left[\frac{g\left(M_{0} M_{0}\right)}{\left[g_{s_{1}}(0,0) g_{s_{2}}(L, L)\right]^{1 / 2}}\right]\right\}$,

where $g\left(M_{0} M_{0}\right)$ is the Green's function of the whole system at its both extremities $M_{0}=\{0, L\}$, whereas $g_{s_{1}}(0,0)$ and $g_{s_{2}}(L, L)$ are the elements of the Green's functions at the surfaces 0 and $L$ of the two substrates (air).

In a previous work, some of us [47] have shown analytically a relation between the transmission delay time $\tau_{T}$ and the variation of the density of states $\Delta n(\omega)$ for isotropic layered media, namely 

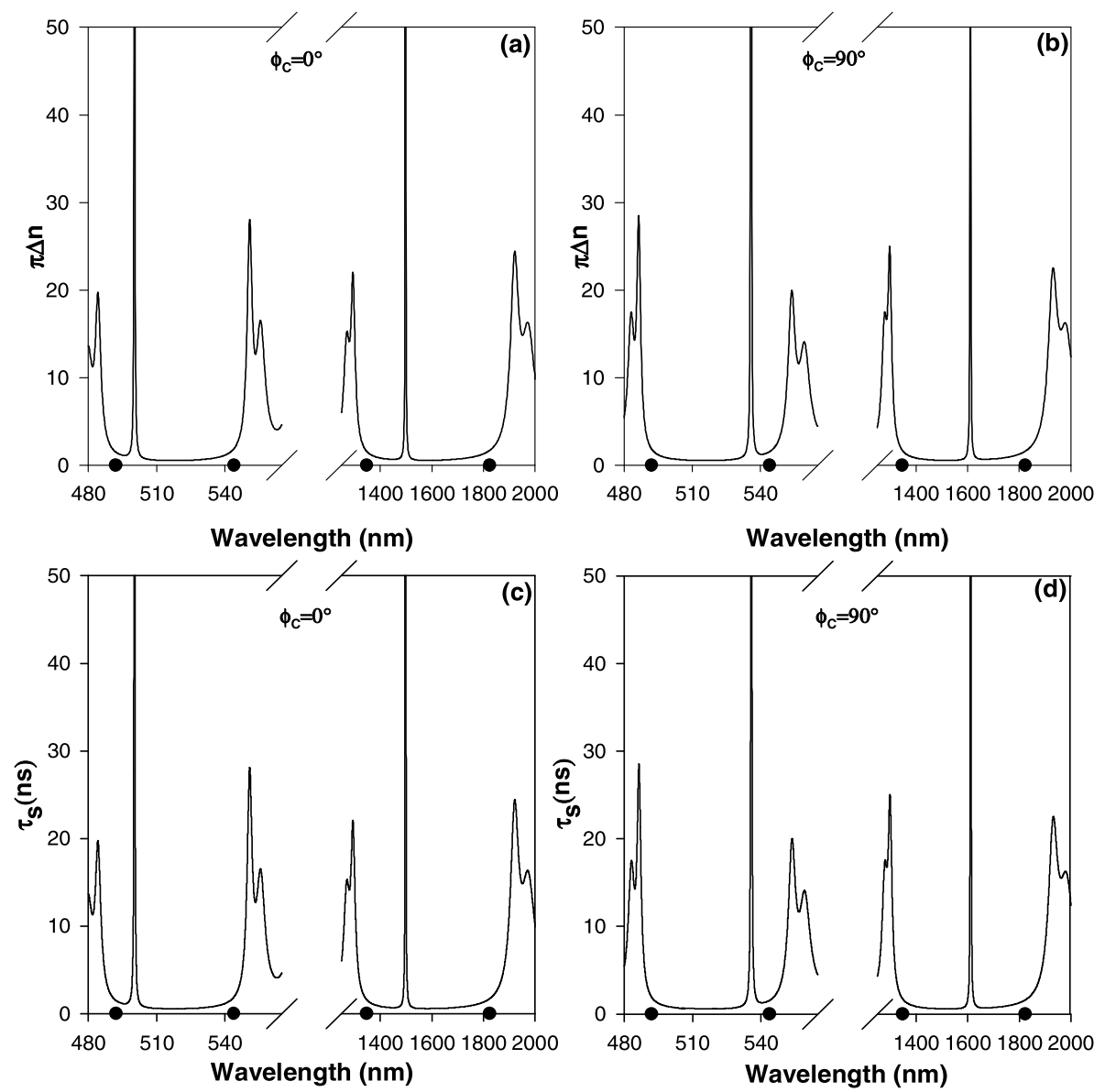


$\phi_{C}=90^{\circ}$ (b, d). The black dots on the $\lambda$-axis represent the edges of the band gaps of the infinite isotropic SL at normal incidence.

$\tau_{T}=\pi \Delta n(\omega)$. In the case of anisotropic media, the expressions of the different delay times (Eq. (11)) and $\Delta n(\omega)$ (Eq. (12)) are more complicated. Therefore, we limit ourselves to a numerical comparison.

\section{Numerical results and discussion}

The perfect photonic crystal $s_{1} /(A B)^{N} / s_{2}$ consists of two alternating isotropic media: $\mathrm{TiO}_{2}$ (layer $A$ ) and $\mathrm{SiO}_{2}$ (layer $B$ ). The refractive indices of these materials are, respectively, $n_{A}=2.35$ and $n_{B}=1.46$. The thicknesses $d_{A}$ and $d_{B}$ of these layers are chosen in accordance with the quarter wave stack condition, i.e., $n_{A} d_{A}=$ $n_{B} d_{B}=\frac{\lambda_{0}}{4}$ where $\lambda_{0}=1550 \mathrm{~nm}$, which leads to $d_{A}=164.89 \mathrm{~nm}$ and $d_{B}=265.41 \mathrm{~nm}$, respectively. $\lambda_{0}=1550 \mathrm{~nm}$ is chosen for its use in optical communications. The number $N$ of the $A B$ bilayers (periods) of the PC is chosen equal to $N=6$, for which the PBGs are well defined. Both substrates surrounding the PC are simply assumed to be air. The periodicity of the perfect SL is broken by inserting the defect layer $C$ in the center of the structure as depicted in Fig. 1. The defect layer is made of an anisotropic dielectric material: SbSI, with its principal optical indices [39]: $n_{x}^{(C)}=2.7, n_{y}^{(C)}=3.2, n_{z}^{(C)}=3.8$. The orientation of the principal axes of the layer $C$ is characterized by the azimuthal angle $\phi_{C}$, as illustrated in Fig. 1, and the thickness of this layer is chosen equal to $d_{C}=263.50 \mathrm{~nm}$, for which the defect modes are well defined in PBGs. In most of our numerical results, we study the behavior of the optical modes in a defective 1D PC when the incoming light has $S$ or $P$ polarization. After multiple reflection and transmission in the anisotropic layer $C$, the incoming wave produces two reflected and two transmitted waves with $S$ and $P$ polarizations. For special cases when the principal axes of the defect layer are parallel or perpendicular to the fixed axes, the $S$ and $P$ polarized waves remain uncoupled [45]. Therefore, a single incident $S$ or $P$ polarized plane wave generates a single reflected $R_{S}$ or $R_{P}$ polarized plane wave and a single transmitted $T_{S}$ or $T_{P}$ polarized plane wave, respectively.

We display in Fig. 2 the transmittances $T_{P}$ (Fig. 2(a), (b)) and $T_{S}$ (Fig. 2(c), (d)) of the optical modes as a function of the wavelength $\lambda$ for a perfect PC (solid lines) and a defective PC (dashed lines). In this illustration, we assume that the incident electromagnetic wave is launched normally $\left(\theta_{i}=0^{\circ}\right)$ to the $\mathrm{SL}$, and the principal axes of the defect layer $C$ are parallel $\phi_{C}=0^{\circ}$ (Fig. 2(a), (c)), or perpendicular $\phi_{C}=90^{\circ}$ (Fig. 2(b), (d)) to the fixed axes of the coordinates system $(X Y Z)$. It is shown that for these two values of the azimuthal angle $\phi_{C}$, the defect modes appear inside the PBGs of the transmission spectrum. The localized modes in the electromagnetic forbidden bands of the structure arise due to the constructive interference of incident and reflected waves in the defect layer. From the analysis of Fig. 2, it can be observed that when the principal axes of anisotropic defect layer were parallel to the fixed axes of the coordinates system, the defect modes (dashed curves) are localized at $\lambda_{P_{0}}^{V I S}=535.81 \mathrm{~nm}, \lambda_{P_{0}}^{I R}=1611.47 \mathrm{~nm}$ in the visible (VIS) and infrared (IR) regions, respectively, for transmission spectrum of $P$-polarized wave (Fig. 2(a)). Moreover, when the azimuthal angle of the layer $C$ is equal to $90^{\circ}$, both modes shift to shorter wavelengths and appear respectively at $\lambda_{P_{90}}^{V I S}=500.22 \mathrm{~nm}$ and $\lambda_{P_{90}}^{I R}=1497.83 \mathrm{~nm}$ (Fig. 2(b)). The comparison of the results for $S$ (Fig. 2(c, d)) and $P$ (Fig. 2(a, b)) polarized incident waves, 

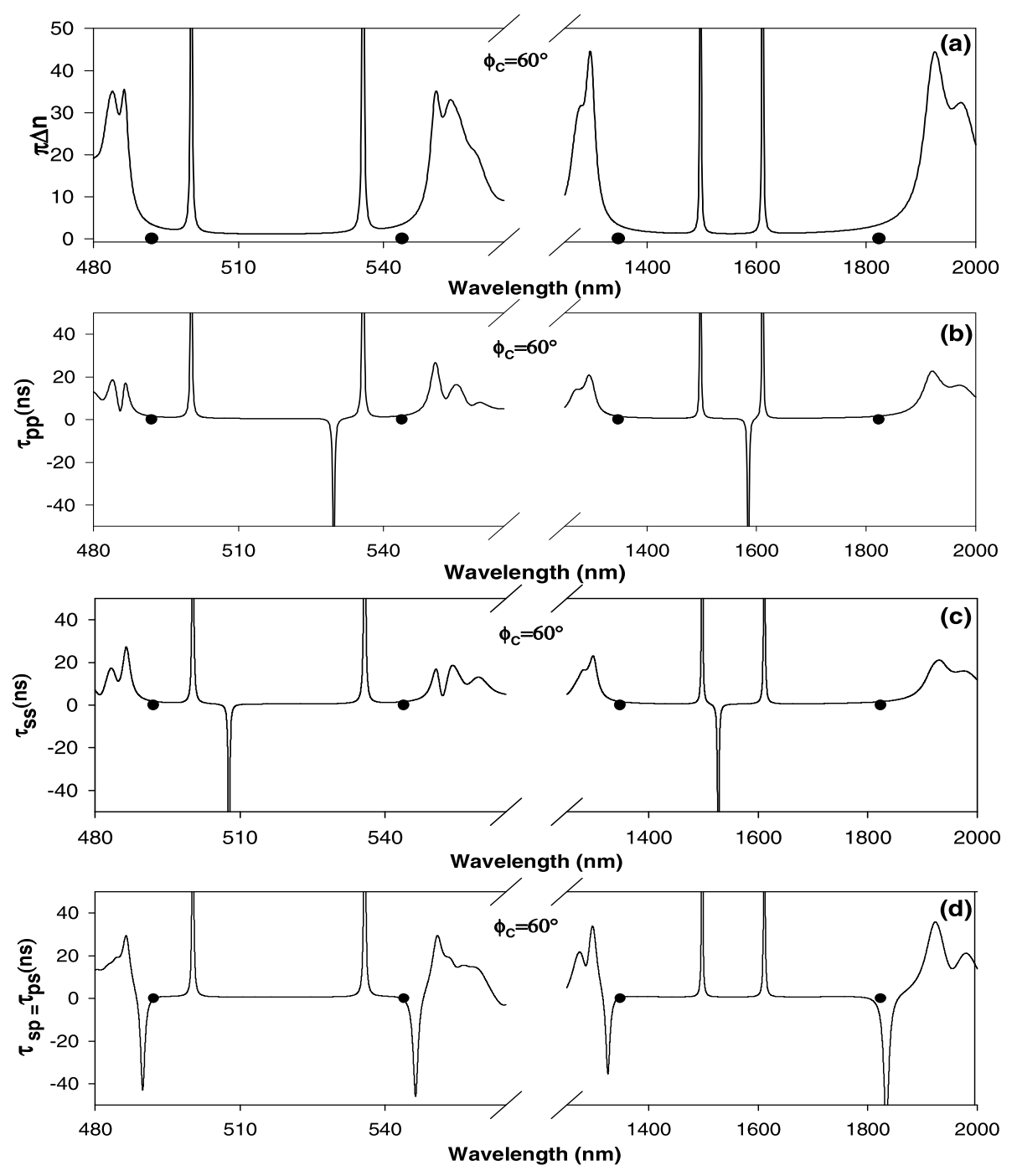

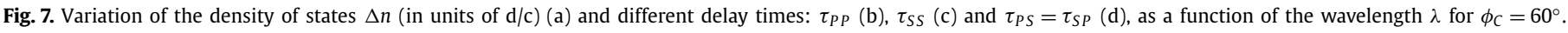
The black dots on the $\lambda$-axis represent the edges of band gaps of the infinite isotropic SL and for normal incidence.

shows that the $S$-polarized defect modes for $\phi_{C}=0^{\circ}$ have the same behavior as the $P$-polarized defect modes when $\phi_{C}=90^{\circ}$, i.e., $\lambda_{S_{0}}^{V I S}=\lambda_{P_{90}}^{V I S}$ and $\lambda_{S_{0}}^{I R}=\lambda_{P_{90}}^{I R}$. Moreover, the same behavior of the localized modes in PBGs has been observed when the principal axes of defect layer are perpendicular to the fixed axes and the incoming light has $S$ polarization, i.e., $\lambda_{S_{90}}^{V I S}=\lambda_{P_{0}}^{V I S}$ and $\lambda_{S_{90}}^{I R}=\lambda_{P_{0}}^{I R}$. These results are in good accordance with those presented in Ref. [48]. In addition, the transmission amplitudes of these peaks reach approximately unity for $\phi_{C}=0^{\circ}$ and $\phi_{C}=90^{\circ}$. The black dots on the $\lambda$-axis indicate the limits of the band gaps of the infinite isotropic SL. These findings clearly show that the orientation of the principal axes of the anisotropic defect layer changes the properties of the defect modes within the VIS and IR regions band gaps. In order to understand the spatial distribution of the $S$-polarized defect modes labeled 1, 2 in Fig. 2(c) along the $Z$-axis of the SL, we have plotted in Fig. 3 the local density of states versus the reduced position $Z / d$ for the azimuthal angle $\phi_{C}=0^{\circ}$ of the defect layer. This quantity reflects the spatial behavior of the square modulus of the electric field inside the structure. Figs. 3(a) and (b) show a strong localization of the defect modes 1 and 2 in the vicinity of the defect layer $C$ and a decaying behavior on both sides of the interfaces of this layer. Similar behavior of the localized mode can be observed for other orientations of the principal axes of the defect layer and also for $P$-polarized waves.
In the following, we consider (Fig. 4) the effect of the incidence angle on the optical properties of the defect modes within the forbidden band gaps in the VIS and IR regions for $\phi_{C}=0^{\circ}$ and $\phi_{C}=90^{\circ}$. Fig. 4 gives the dependence of the peak wavelengths on the angle of incidence, when the input light has $P$ (Fig. 4(a, b)) or $S$ (Fig. 4(c, d)) polarization. One can notice that when the incidence angle increases, the position of $P$ and $S$ polarized defect modes shift towards the lower wavelengths inside the PBGs (hatched areas) as illustrated in Fig. 4. In addition, when the incidence angle is greater than $35^{\circ}$, the defect branch within the first gap for $P$ polarization and $\phi_{C}=0^{\circ}$ (Fig. 4(a)) and for $S$ polarization and $\phi_{C}=90^{\circ}$ (Fig. 4(d)), disappear at the higher edge of the forbidden gap and falls in the allowed band. Furthermore, the transmission amplitudes of these modes remain almost unity whatever the incidence angle for both cases $\left(\phi_{C}=0^{\circ}\right.$ and $\phi_{C}=90^{\circ}$ ).

Till now, we have depicted only the properties of the defect modes in the transmission spectrum of the defective 1D PC when the orientation of the principal axes of defect layer are parallel or perpendicular with respect to the laboratory axes. Let us now consider the effect of other orientations of the axes of the layer $C$ on the behavior of the defect mode when the incident electromagnetic wave is launched normally to the SL. We present in Fig. 5(a, b), the evolution of the transmission spectra $T_{S S}$ and $T_{P S}$ 

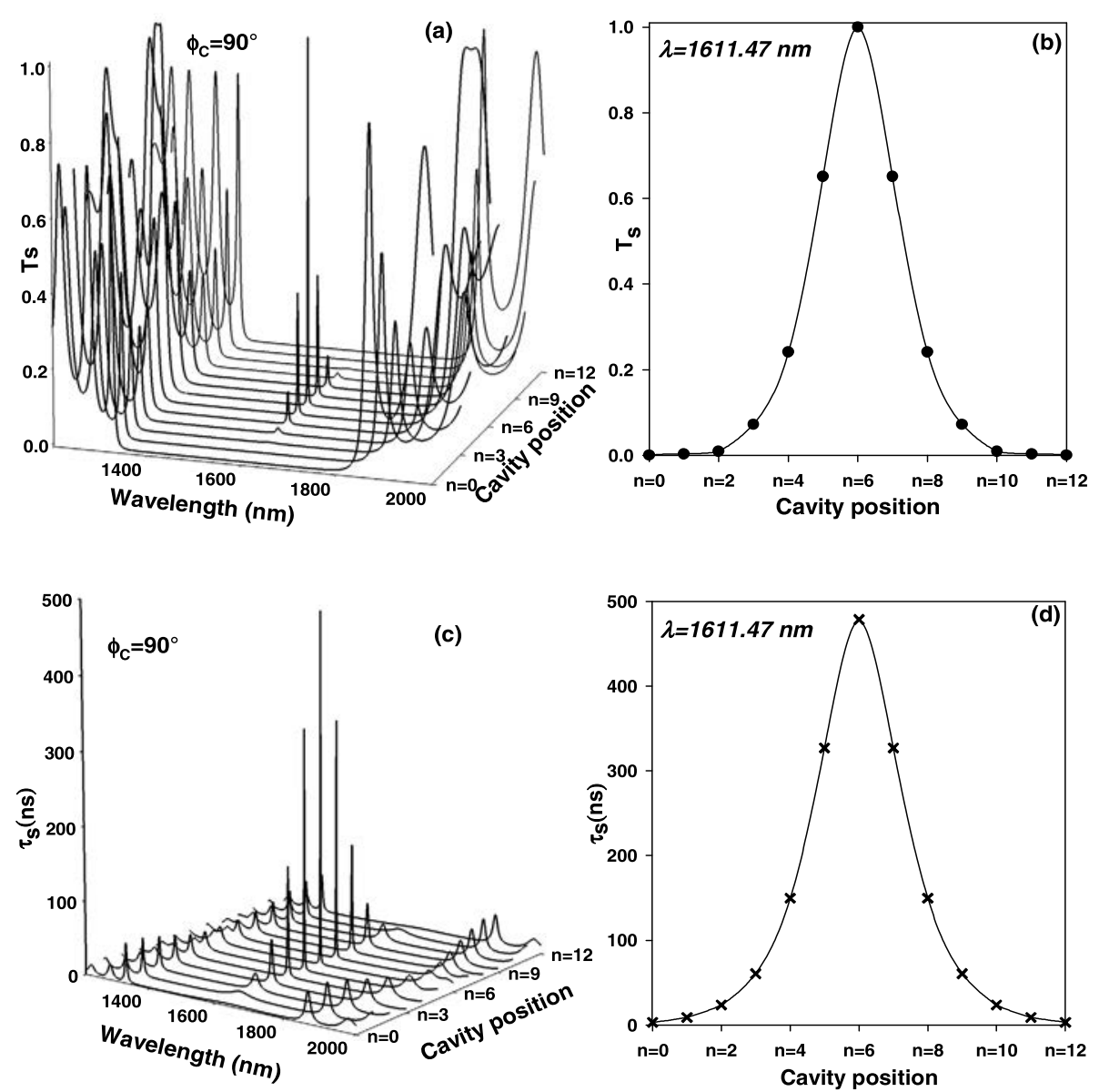

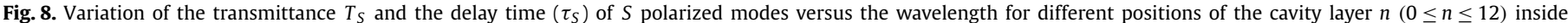

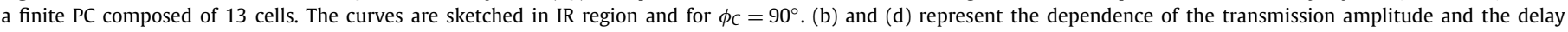
time of the defect modes as a function of the defect cavity position $n$.

of $S$-polarized wave as a function of the wavelength $\lambda$ for the azimuthal angle $\phi_{C}=60^{\circ}$. It is clear that the birefringence of the defect layer has a strong impact on the number and the magnitudes of the defect modes inside the PBGs in the VIS and IR regions. For this azimuthal angle $\left(\phi_{C}=60^{\circ}\right)$ of the layer $C$, two defect modes appear inside each PBGs as every incident plane wave with $S$ or $P$ polarization generates two reflected and two transmitted plane waves containing both $S$ and $P$ polarized plane waves. These two propagating waves are coupled, thus each mode is composed of two components of the transmission ( $T_{S S}$ and $T_{P S}$ ) spectrum for the same wavelength. These results correlated with the results in our previous work [33], when the PC is composed only by anisotropic media.

Moreover, the intensities of the defect modes depend strongly on the azimuthal angle of the anisotropic defect cavity as illustrated in Fig. 5(c, d), where we have plotted the dependence of peak heights $T_{S S}$ (Fig. 5(c)) and $T_{P S}$ (Fig. 5(d)) of the modes labeled 1 and 2 in Figs. 5(a) and (b) versus the azimuthal angle $\phi_{C}$. The results of Fig. 5(c) show that by adjusting $\phi_{C}$, the intensities of the defect modes exhibit different behaviors inside the PBGs of the structure. Indeed, by increasing $\phi_{C}$ from $0^{\circ}$ to $90^{\circ}$, the transmission amplitudes of the mode 2 (indicated by the black dots) increase from 0 to 1 , while the amplitudes of the mode 1 (indicated by black triangles) decrease from 1 to 0 . However, the transmitted $P$-polarized modes (Fig. 5(d)), exhibit similar behavior. Their intensities gradually increase by rotating the azimuthal angle of the defect layer, reach a maximum value around $\phi_{C}=45^{\circ}$ and then decrease to vanish for $\phi_{C}=90^{\circ}$. Also, both transmitted
$P$ - and $S$-polarized defect modes have identical magnitudes $(0.25)$ around $\phi_{C}=45^{\circ}$.

The Green's function method enables us to derive other physical properties of the composite system under study, through the analysis of the transmission delay time $\tau$ and the total density of states $\Delta n$. The delay time describes the time needed for photons to complete the transmission process and provides information about the interaction of an incident wave with the modes confined in the anisotropic defect layer. In the following, we study these two quantities and give a comparison between the total DOS and the delay time of the optical waves. We display in Fig. 6, the variation of DOS $(\pi \Delta n)$ (Fig. 6(a, b)) and the delay time $\tau$ (Fig. 6(c, d)) as a function of the wavelength for $S$-polarized incident wave and when the principal axes of defect layer are parallel or perpendicular to the fixed ones. It is clear that the delay time of the transmitted peaks in the VIS and IR regions are very sensitive to the azimuthal angle of the anisotropic defect layer. The frequencies of the defect modes obtained from the total DOS (Fig. 6(a, b)) coincide with those given by the delay time (Fig. 6(c, d)) and the transmission coefficient (see Fig. 3(c, d)). In addition, the delay time $\tau_{s}$ exhibits the same behavior as the DOS $(\pi \Delta n)$ for $\phi_{C}=0^{\circ}$ (Figs. 6(c) and (a)) and for $\phi_{C}=90^{\circ}$ (Figs. 6(d) and (b)). For these particular orientations of the principal axes of the defect layer, the density of states is directly proportional to the transmission delay time, i.e., $\tau_{s}=\pi \Delta n(\omega)$, in accordance with the results demonstrated for isotropic PC [47]. These results remain also valid for $P$-polarized waves. However, for $\phi_{C} \neq 0^{\circ}$ and $\phi_{C} \neq 90^{\circ}$, different transmission delay times $\left(\tau_{s s}, \tau_{p s}\right)$ and $\left(\tau_{p p}, \tau_{s p}\right)$ for $S$ and $P$ transmitted polarized waves, respectively, show different behaviors in comparison 
with the DOS as it is illustrated in Fig. 7. One can notice that the delay times spectrum for both $S$ and $P$ transmitted polarized waves show negatives peaks for some wavelengths in the VIS and IR regions. We have checked that the corresponding transmission amplitudes reach almost zero as a consequence of a destructive interference between $S$ and $P$ waves inside the anisotropic defect layer. The negative peaks in $\tau_{p p}$ (Fig. 7(b)) and $\tau_{s s}$ (Fig. 7(c)) spectra are localized between the two defect modes in both VIS and IR regions, whereas those in $\tau_{s p}$ and $\tau_{p s}$ (Fig. 7(d)) appear at the vicinities of the PBG edges. It is worth mentioning that the negative delay times in 1D systems are generally observed by taking into account the absorption in these systems [36,49]. However, in the present system, the negative delta peaks are intrinsically broadened. Moreover, because of the symmetry of the structure, the delay time $\tau_{s p}$ presents the same behavior as $\tau_{p s}$. Indeed, rendering the structure asymmetric, by moving for example the cavity layer $C$ inside the PC, gives rise to $\tau_{p s} \neq \tau_{s p}$. Let us notice that the existence of transmission zeros in one of the two components of the transmission, may give rise also to Fano and electromagnetic induced transparency resonances [35] even though these properties have not been explored here and postponed to a future work.

To widen the scope of our findings, we have also investigated the effect of the position of the cavity layer within the PC on the optical properties of defect modes in the PBGs of the structure. We have plotted in Fig. 8, the transmittance Fig. 8(a, b) and the delay time Fig. 8(c, d) of the localized modes in the IR region for different positions of the cavity layer within the PC and for $\phi_{C}=90^{\circ}$. The PC is composed of 13 cells and the cavity layer can be inserted at different positions labeled $n$ inside the PC $(0 \leq n \leq 12)$. The transmission spectra are displayed for S-polarized waves. One can notice that the transmittance and the delay time of the defect modes depend strongly on the position of the cavity layer within the finite SL. The magnitudes of the filtered modes in the PBGs, reach their maximum value when the defect cavity is inserted in the middle of the structure (i.e., $n=6$ ) and decreases gradually when the defect layer is moved far from the middle of the PC, as shown in Figs. 8(b) and (d). The transmittance and the delay time almost vanish when the cavity layer is placed at both extremities of the structure. These results remain also valid for other orientations of the principal axes of the defect layer and for $P$-polarized waves.

\section{Conclusion}

In summary, we have investigated theoretically the effect of an anisotropic defect cavity on the optical transmission properties in one-dimensional isotropic photonic crystal. This study is performed by means of the Green's function approach, through an investigation of the transmission coefficients, the delay times and the DOS for electromagnetic modes in the finite structure. The transmittance and the delay times are discussed as a function of the azimuthal angle and the position of the anisotropic defect cavity within the PC. We have presented, for the first time to our knowledge, a comparison between the total DOS and different transmission delay times in such systems. We have shown that the delay time reproduces exactly the DOS when the principal axes of the defect layer are parallel or perpendicular to the laboratory axes. However, far from these directions, the birefringence of the cavity layer induces, in addition to mode conversion, transmission zeros in one of the two components of the transmission, for some wavelengths in the VIS and IR regions, giving rise to negative delta peaks in the delay times. These properties are without analogue in their counterpart isotropic defect layers. This structure can be of potential interest in the fields of photonics and optoelectronics as a high-precision optical filter.

\section{Acknowledgements}

This research did not receive any specific grant from funding agencies in the public, commercial, or not-for-profit sectors.

\section{References}

[1] E. Yablonovitch, Phys. Rev. Lett. 58 (1987) 2059-2062.

[2] S. John, Phys. Rev. Lett. 58 (1987) 2486-2489.

[3] Y. Fink, J.N. Winn, S. Fan, C. Chen, J. Michel, J.D. Joannopoulos, E.L. Thomas, Science 282 (1998) 1679.

[4] M.S. Vitiello, M. Nobile, A. Ronzani, A. Tredicucci, F. Castellano, V. Talora, L. Li, E.H. Linfield, A.G. Davies, Nat. Commun. 5 (2014) 5884.

[5] Y. Liang, C. Peng, K. Ishizaki, S. Iwahashi, K. Sakai, Y. Tanaka, K. Kitamura, S. Noda, Opt. Express 21 (2013) 565-580.

[6] C. Wang, C.Z. Zhou, Z.Y. Li, Opt. Express 19 (2011) 26948-26955.

[7] Y. Zhang, D. Li, C. Zeng, Z. Huang, Y. Wang, Q. Huang, Y. Wu, J. Yu, J. Xia, Opt. Lett. 39 (2014) 1370-1373.

[8] J. Rosenkrantz de Lasson, P.T. Kristensen, J. Mork, N. Gregersen, Opt. Lett. 40 (2015) 5790-5793.

[9] A.E. Erol, H.S. Sozuer, Opt. Express 23 (2015) 32690-32695.

[10] J. Liu, P.D. Han, G.J. Qiao, J.F. Yang, J. Intense Pulsed Lasers Appl. Adv. Phys. 1 (2011) 69.

[11] A. Mir, A. Akjouj, E.H. El Boudouti, B. Djafari-Rouhani, L. Dobrzynski, Vacuum 63 (2001) 197.

[12] M. Upadhyay, S.K. Awasthi, S.K. Srivastava, S.P. Ojha, Prog. Electromagn. Res. 25 (2012) 211

[13] N. Ouchani, D. Bria, B. Djafari Rouhani, A. Nougaoui, J. Phys. Condens. Matter 21 (2009) 485401-485410.

[14] I. Abdulhalim, Opt. Commun. 174 (2000) 43-50.

[15] A. Bouzidi, D. Bria, A. Akjouj, Y. Pennec, B. Djafari-Rouhani, J. Phys. D, Appl. Phys. 48 (2015) 495102

[16] J.-J. Wu, J.-X. Gao, Mater. Chem. Phys. 171 (2016) 91-96.

[17] K. Inoue, K. Ohtaka, Photonic Crystals: Physics, Fabrication and Applications, Springer-Verlag, New York, 2004.

[18] Y.J. Xiang, X.Y. Dai, S.C. Wen, D.Y. Fan, Opt. Lett. 33 (2008) 1255-1257.

[19] N.N. Dadoenkova, A.E. Zabolotin, I.L. Lyubchanskii, Y.P. Lee, Th. Rasing, J. Appl. Phys. 108 (2010) 093117.

[20] C.-Y. Wu, Y.-H. Zou, I. Timofeev, Y.-T. Lin, V.Y. Zyryanov, J.-S. Hsu, W. Lee, Opt. Express 19 (2011) 7349-7355.

[21] H.T. Wang, I.V. Timofeev, K. Chang, V.Y. Zyryanov, W. Lee, Opt. Express 22 (2014) 15097

[22] K. Jamshidi-Ghaleh, B. Kazempour, J. Appl. Opt. 55 (2016) 4350-4356.

[23] W. Zhang, P. Han, A. Lan, Y. Li, X. Zhang, Physica E 44 (2012) 813-815.

[24] T. Tang, W. Liu, X. Gao, X. He, J. Yang, Opt. Laser Technol. 43 (2011) 1016-1019.

[25] L. Penninck, P. De Visschere, J. Beeckman, K. Neyts, Opt. Express 19 (2011) 18558.

[26] I.V. Soboleva, E.M. Murchikova, A.A. Fedyanin, O.A. Aktsipetrov, Appl. Phys. Lett. 87 (2005) 241110.

[27] V.A. Belyakov, S.V. Semenov, J. Exp. Theor. Phys. 118 (2014) 798.

[28] A.H. Gevorgyan, A. Kocharian, G.A. Vardanyan, Opt. Commun. 259 (2006) 455-464.

[29] Y.C. Yang, C.S. Kee, J.E. Kim, H.Y. Park, J.C. Lee, Y.J. Jeon, Phys. Rev. E 60 (1999) 6852.

[30] A. Lakhtakia, V.C. Venugopal, M.W. McCall, Opt. Commun. 177 (2000) 57-68.

[31] A.H. Gevorgyan, M.Z. Harutyunyan, Phys. Rev. E 76 (2007) 031701.

[32] A.H. Gevorgyan, M.Z. Harutyunyan, J. Mod. Opt. 56 (2009) 1163-1173.

[33] N. Ouchani, D. Bria, B. Djafari-Rouhani, A. Nougaoui, J. Appl. Phys. 106 (2009) 113107.

[34] K. Zhang, J. Fu, L.Y. Xiao, Q. Wu, L.W. Li, J. Appl. Phys. 113 (2013) 084908.

[35] W. Zhou, D. Zhao, Y.-C. Shuai, H. Yang, S. Chuwongin, A. Chadha, J.-H. Seo, K.X. Wang, V. Liu, Z. Ma, S. Fan, Prog. Quantum Electron. 38 (2014) 1-74.

[36] E.H. El Boudouti, N. Fettouhi, A. Akjouj, B. Djafari-Rouhani, A. Mir, J.O. Vasseur, L. Dobrzynski, J. Zemmouri, J. Appl. Phys. 95 (2004) 1102.

[37] H. Goldstein, Classical Mechanics, Addison-Wesley, 1957, pp. 107-109.

[38] G.D. Landry, T.A. Maldonado, J. Opt. Soc. Am. A 12 (1995) 2048-2063.

[39] P. Yeh, Optical Waves in Layered Media, Wiley, 1988, pp. 201-253.

[40] P. Yeh, A. Yariv, Optical Waves in Crystals: Propagation and Control of Laser Radiation, John Wiley \& Sons, 2002.

[41] L. Dobrzynski, E.H. El Boudouti, A. Akjouj, Y. Pennec, H. Al-Wahsh, G. Lévêque, B. Djafari-Rouhani, Phononics, Elsevier, 2017.

[42] V.R. Velasco, F. Garcia-Moliner, H. Rodriguez-Coppola, R. Pétrez-Alvarez, Phys. Scr. 41 (1990) 375-382.

[43] E.H. El Boudouti, B. Djafari-Rouhani, A. Akjouj, L. Dobrzynski, Phys. Rev. B 54 (1996) 14728

[44] L. Dobrzynski, Surf. Sci. 180 (1987) 489-504 
[45] N. Ouchani, D. Bria, B. Djafari-Rouhani, A. Nougaoui, J. Opt. Soc. Am. A 24 (2007) 2710-2718

[46] B. Djafari-Rouhani, L. Dobrzynski, J. Phys. Condens. Matter 5 (1993) 139.

[47] M.L.H. Lahlaouti, A. Akjouj, B. Djafari-Rouhani, L. Dobrzynski, M. Hammouchi, E.H. El Boudouti, A. Nougaoui, B. Kharbouch, Phys. Rev. B 63 (2001) 035312.
48] V.I. Fesenko, I.A. Sukhoivanov, S.N. Shulǵa, J.A. Andrade Lucio, Advances in Photonic Crystals, Vittorio M.N. Passaro, 2013, pp. 79-105.

[49] Y.El. Hassouani, E.H. El Boudouti, B. Djafari-Rouhani, H. Aynaou, Phys. Rev. B 78 (2008) 174306 